\title{
Single photon sources for quantum radiometry: a brief review about the current state-of-the-art
}

\author{
Stefan Kück ${ }^{1,2}$ (1) Marco López ${ }^{1} \cdot$ Helmuth Hofer ${ }^{1} \cdot$ Hristina Georgieva $^{1} \cdot$ Justus Christinck ${ }^{1,2}$ - Beatrice Rodiek ${ }^{1}$. \\ Geiland Porrovecchio ${ }^{3}$. Marek Šmid ${ }^{3}$. Stephan Götzinger ${ }^{4,5}$. Christoph Becher ${ }^{6}$. Philipp Fuchs ${ }^{6}$. Pietro Lombardi ${ }^{7}$. \\ Costanza Toninelli ${ }^{7} \cdot$ Marco Trapuzzano $^{8} \cdot$ Maja Colautti $^{9} \cdot$ Giancarlo Margheri $^{10} \cdot$ Ivo Pietro Degiovanni $^{11}$. \\ Paolo Traina ${ }^{11} \cdot$ Sven Rodt $^{12} \cdot$ Stephan Reitzenstein ${ }^{12}$
}

Received: 10 October 2021 / Accepted: 13 December 2021 / Published online: 23 January 2022

(c) The Author(s) 2022

\begin{abstract}
Single-photon sources have a variety of applications. One of these is quantum radiometry, which is reported on in this paper in the form of an overview, specifically of the current state of the art in the application of deterministic single photon sources to the calibration of single photon detectors. To optimize single-photon sources for this purpose, extensive research is currently carried out at the European National Metrology Institutes (NMIs), in collaboration with partners from universities. Single-photon sources of different types are currently under investigation, including sources based on defect centres in (nano-)diamonds, on molecules and on semiconductor quantum dots. We will present, summarise, and compare the current results obtained at European NMIs for single-photon sources in terms of photon flux, single-photon purity, and spectral power distribution as well as the results of single-photon detector calibrations carried out with this type of light sources.
\end{abstract}

\section{Introduction}

This paper deals with the use of single-photon sources in the field of quantum radiometry, in particular for the calibration of single-photon detectors. A perfect single-photon source is a light source that emits light as single photons, i.e., two or more photons are never emitted simultaneously. This distinguishes it from coherent light sources (lasers) and thermal light sources such as incandescent light bulbs. Single photons can be realized by single atoms, ions, molecules, colour

Stefan Kück

stefan.kueck@ptb.de

1 Physikalisch-Technische Bundesanstalt (PTB), Braunschweig, Germany

2 Laboratory for Emerging Nanometrology, Braunschweig, Germany

3 Český Metrologický Institut (CMI), Okruzni 31, 63800 Brno, Czech Republic

4 Department of Physics, Graduate School in Advanced Optical Technologies (SAOT), Friedrich Alexander University (FAU) Erlangen-Nürnberg, 91052 Erlangen, Germany

5 Max Planck Institute for the Science of Light, 91058 Erlangen, Germany centres and quantum dots, i.e., in systems, where per se the system has to be re-excited before the emission of a second photon. Single-photon sources based on this principle are called deterministic single photon sources and they could produce so-called "photons on demand" in the limit to perfect emission and collection efficiency. Another possible way to generate single photons is the use of spontaneous parametric down-conversion. In this case, pairs of single photons are produced via a nonlinear process; the detection of one photon in one beam path heralds a single photon in the

6 Fachrichtung Physik, Universität des Saarlandes, Campus E2.6, 66123 Saarbrücken, Germany

7 Istituto Nazionale di Ottica (CNR-INO), Florence, Italy

8 Università degli Studi di Firenze, Florence, Italy

9 LENS, Università degli Studi di Firenze, Florence, Italy

10 Istituto dei Sistemi Complessi (CNR-ISC), Florence, Italy

11 Istituto Nazionale di Ricerca Metrologica (INRiM), Torino, Italy

12 Institut für Festkörperphysik, Technische Universität Berlin, Hardenbergstraße 36, 10623 Berlin, Germany 
other beam path. These sources are called "heralded singlephoton sources" or "probabilistic single-photon sources". Vast literature can be found on single-photon sources, an overview is, e.g., given in [1, 2]. Thus, in general, a single-photon source is a one-photon number state generator. Therefore, the field of applications for single-photon sources is wide [1]. Among the most prominent are quantum key distribution, quantum communication, quantum computing and quantum metrology [3]. However, so far single-photon sources compete in all these fields with highly developed laser sources, so that a quantum advantage of the so-called "quantum-enhancement" still has to be proven to be applicable in real world applications. One specific field, in which this advantage has been proven is quantum radiometry [4]. Quantum radiometry means, within the context of this paper, the use of single-photon sources in radiometric applications. In a wider sense, radiometric applications for single-photon sources are:

Standard photon sources: as there are already the blackbody radiator and the synchrotron radiation source with their calculable photon flux, also single-photon sources have the potential to become a new type of standard photon source [5-7]. Consider a device (in this case a perfect single-photon source), which emits under excitation with a pulsed laser operating with a repetition rate $f$ exactly one photon per excitation pulse. The optical radiant flux $\Phi$ of such a device would be exactly given by $\Phi=f h c / \lambda$, where $f$ is the repetition rate of the excitation laser, $h$ is the Planck constant, $c$ is the speed of light and $\lambda$ the wavelength of the emitted radiation. Since $c$ and $h$ have no uncertainty, and the frequency and wavelength can be measured with uncertainties in the $10^{-17}$ and $10^{-12}$ range, respectively, the optical radiant flux could be determined with an unprecedented accuracy, in particular far below the current state-of-the-art achieved using the cryogenic radiometer, which has uncertainties in the $10^{-5}$ range [8]. However, this requires a perfect source, i.e., a source with a quantum efficiency of $100 \%$ (i.e., each excitation leads to an emission of a photon), a perfect purity of the single-photon emission, i.e., $g^{(2)}(0)=0$, and a collection efficiency of the emitted radiation of $100 \%$. On the other hand, as will be described in detail within this paper, single-photon sources are ideal sources for the calibration of single-photon detectors, because the necessary correction for the photon statistics when using a laser source is completely eliminated or at least significantly diminished for imperfect single-photon sources (see $[9,10])$. This combined "dead time and photon statistics effect" of, e.g., a SPAD detector, is described in Sect. 2.1.

Sub-shot noise metrology is another possible application of single-photon sources in quantum radiometry: it exploits them to carry out measurements below the standard quantum limit. For this, efficient, nearly perfect sources have to be used. In [11], Chu et al. showed in principle the quantum advantage, general ideas are given in [12]. Other, relevant applications for single-photon sources are in the field of quantum imaging [13], both for achieving sub-shot noise imaging $[14,15]$ as well as for super-resolution [16, 17]. Even quantum illumination [18, 19], quantum sensing $[20,21]$ and quantum reading $[22,23]$ are expected to take advantage of efficient single-photon sources.

"Quantum Candela": a realization of the base unit candela using quanta, i.e., countable photons, is intriguing. Therefore, in the mise-en-pratique for the candela [7], the possibility to realize the candela exploiting (single) photons is explicitly stated, thus enabling photometric, radiometric, and their derived quantities to be expressed in terms of photonnumbers and photon number-based quantities.

\section{Detector calibration with single-photon sources}

\subsection{Single-photon detectors}

In contrast to single-photon sources, single-photon detectors are already quite mature and widely commercially available, e.g.: the single-photon avalanche diode (SPAD), the transition edge sensor (TES), and the superconducting nanowire single-photon detector (SNSPD). Medicine, biology, astrophysics, emerging fields like quantum cryptography and quantum computing as well as scientific research in experimental quantum optics and quantum physics are their main fields of application, i.e., wherever low photon fluxes need to be measured. Detailed descriptions of different types of detectors, operation modes and metrological aspects can be found in $[1,2,4,24]$. In this paper, we focus on the application of single-photon sources for the detection efficiency calibration of SPAD detectors.

There are two factors that need to be considered for SPAD detectors: First, they are not photon-number resolving and thus produce at most one 'click' in response to a pulse with one or more photons; second, they have a dead-time when they are not active after a detection. The use of a Poissonian source (i.e. an attenuated laser) yields both multi-photon pulses and, for $\mathrm{CW}$ radiation, a variation in photon arrival times. These factors complicate the calibration of SPADS with attenuated laser light. This has been studied in detail for Si-SPAD detectors by López et al. [9] and Georgieva et al. [25].Photons arriving individually with a time interval longer than the dead time (typically between 10 and $100 \mathrm{~ns}$ for SI-SPADs) can be fully detected by the Si-SPAD, but the same number of photons arriving within one pulse would only allow one detection event, i.e., there is a strong correlation between the dead time and the temporal distribution of photons arriving on the Si-SPAD; the photon number distribution of the photon source used in the calibration 
experiment is therefore important. Hence, the best source for determining the undisturbed, physically relevant detection efficiency would be a single-photon source that delivers photons with a time interval longer than the dead time and at which the photon flux can still be measured with conventional $\mathrm{Si}$ diodes, which act as reference, i.e., they are traceable to the standard for optical radiant flux, the cryogenic radiometer.

\subsection{The nitrogen-vacancy centre in diamond as single-photon source}

The nitrogen vacancy centre (NV-centre) in diamond is one of the most investigated single-photon sources, see e.g. [26-30]. The reason is that the NV-centre is the most naturally occurring colour centre in diamond and its emission is highly efficient also at room temperature. It was also the first commercially available single-photon source [31].

The first comprehensive metrological characterisation of a single-photon source with respect to absolute spectral radiation flux was carried out by Rodiek et al. [32]. In that work, a single-photon source based on an NV centre was metrologically characterised with regard to its most relevant properties. Its photon flux, its spectral radiant flux as well as the purity of the single-photon emission were measured in a traceable manner. The standard measurement uncertainty for the photon flux was about 4\% [33], see also Fig. 1. The total radiant flux was between 55 and $75 \mathrm{fW}$, corresponding to a total photon flux of 190000 photons per second and 260000 photons per second, respectively. The purity of the single photon emission is indicated by the $g^{(2)}(0)$ value, which ranges from 0.10 to 0.23 depending on the excitation power. These values are traceable to the corresponding national standards via an unbroken traceability chain.

(a)

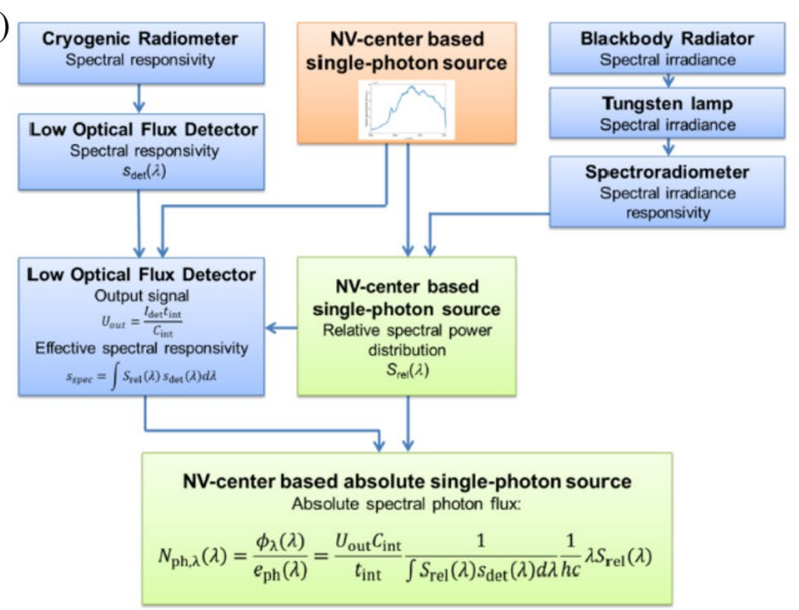

Fig. 1 a Traceability chain for the metrological characterization of a single-photon source in terms of its absolute spectral photon flux, adapted from [32]; b Absolute spectral photon flux of the single-
Even though this source is only suitable for applications to a limited extent due to its broad spectral emission distribution, these first results show the prospects for the general application of single-photon sources, e.g., for the calibration of the detection efficiency of single-photon detectors as well as for the use as a standard photon source in the low photon flux range.

It should be noted that other impurity centres in diamond, which show a more suitable spectral power distribution for calibration purposes, are currently under investigation. These are, e.g., the colours centres based on Silicon [34-37], Germanium [38, 39], Tin [40-43], Fluorine [44, 45], Helium [46] impurities and Lead vacancy centre [47-50], see also the overview article from Moreva et al. [51]. In [52], Vaigu et al. successfully used a single-photon source based on a $\mathrm{SiV}$-centre in nanodiamond with an emission bandwidth of $\Delta \lambda_{\text {FWHM }} \approx 2 \mathrm{~nm}$ for the determination of the detection efficiency of a Si-SPAD detector, however, a direct calibration against a reference detector could not be carried out, because of the low photon rate of approx. 60000 photons per second. Single $\mathrm{SnV}$ centres in high-temperature annealed diamond samples show promising properties for calibration purposes, i. e. a high single photon purity $g^{(2)}(0)<0.05$ limited only by detector dark counts and saturation photon rates of up to 150,000 per second [53]. A further increase in photon count rates might be achieved by combining the $\mathrm{SnV}$ centres with nanophotonic structures, e. g. optical antennas which were recently demonstrated to enhance the saturation emission rates by a factor of 5-10 [54] yielding up to 500,000 photons per second.

(b)

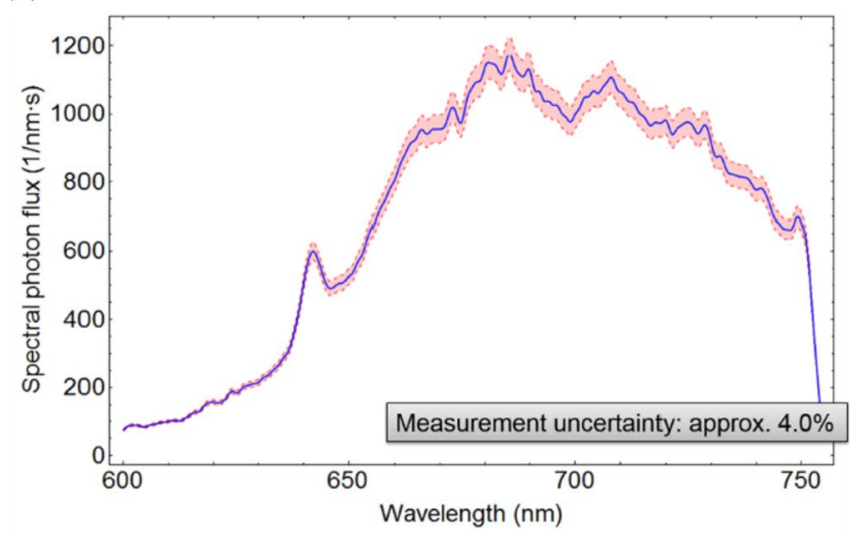

photon source (blue curve). The calculation of the presented measurement uncertainty (red strap) is described in [33]. Taken from [33] 


\subsection{The molecule-based single-photon source for calibration of SPAD detectors}

A better source for the calibration of single-photon detectors is principally a molecule-based single-photon source, which exhibits a narrow-line emission, for two main reasons: spectral corrections are not needed, and the spectral radiant flux is much higher. It should be noted that, for radiometric applications, a linewidth of less than approx. $2 \mathrm{~nm}$ is usually sufficient. Some molecules fulfil these conditions, e.g., terrylene, see e.g. [55], and dibenzoterrylene in anthracene (DBT:Ac), see e.g. [56, 57].

With a DBT:Ac source, Lombardi et al. [58] performed for the first time a direct calibration of a Si-SPAD detector using a continuously operated single-photon source. This molecule emits narrowband photons when cooled to cryogenic temperatures and show high quantum efficiency, photostability and quantum coherence [56, 59, 60], even when embedded in small nanocrystals [61]. The source used for the direct calibration of a Si-SPAD detector against a calibrated analogue Silicon reference detector had a photon flux at the location of the detector of up to $1.32 \times 10^{6}$ photons per second, a value for $g^{(2)}(0)$ (indicating the single-photon purity) $<0.1$ and a spectral bandwidth of $<0.2 \mathrm{~nm}$. This optical radiant flux can still be reasonably measured with conventional silicon photodiodes, see e.g. [62]. Figure 2 summarizes in an artistic manner the properties of the DBT:Ac single-photon source.

The calibration process (for details see [58]), is in this case rather simple, i.e., the substitution method is used. The photon flux from the single-photon source was alternatively

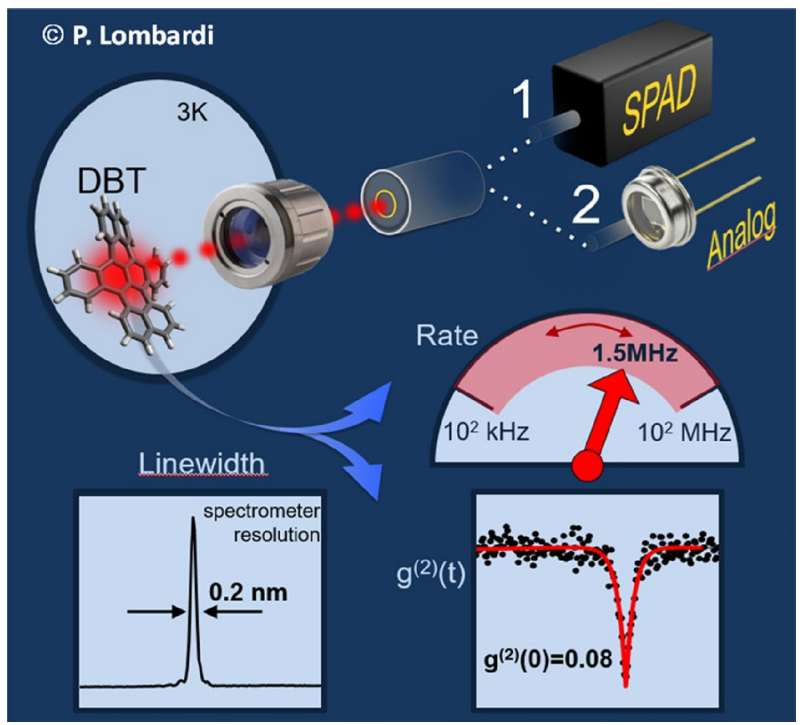

Fig. 2 Artistic summary of the properties of the DBT:Ac singlephoton source used for the calibration of the Si:SPAD detector. (C) P. Lombardi measured with the SPAD detector and with an analogue reference Si detector, which is traceable to the standard for optical radiant flux, i.e., the cryogenic radiometer. Both detectors were equipped with an FC/PC multimode fibre port, so that the output from the fibre coupled single-photon source can be easily measured. The SPAD detection efficiency $\eta_{\mathrm{SPAD}}$ can then be calculated from:

$\eta_{\mathrm{SPAD}}=\frac{N_{\mathrm{SPAD}}}{N_{\mathrm{Ref}}}=\frac{N_{\mathrm{SPAD}}}{\Phi_{\mathrm{s}} E}=\frac{N_{\mathrm{SPAD}}}{I_{\mathrm{f}} / s_{\text {ref }} E}$,

where $N_{\text {SPAD }}$ is the count rate measured with the SPAD detector, $N_{\text {ref }}$ is the photon flux, determined with the reference detector via the measurement of the photocurrent $I_{\mathrm{f}}$, using the known spectral responsivity $s_{\text {ref }}$ and the photon energy $E\left(=2.53 \times 10^{-19} \mathrm{~J}\right)$ for a photon at $\left.785.6 \mathrm{~nm}\right)$. In Fig. $3, \eta_{\mathrm{SPAD}}$, determined as described above, for the SPAD detector is depicted for photon rates between $0.144 \times 10^{6}$ and $1.32 \times 10^{6}$ photons/s, corresponding to a power range between 36.5 and $334 \mathrm{fW}$. It can be seen from the figure that the photon rate approaches the regime where the detector dead time affects the detection efficiency measurement $\eta_{\text {SPAD }}$ [9]. In this respect, a considerable improvement for the calibration process would be an operation of the singlephoton source in pulsed mode while maintaining the high photon flux. The standard uncertainty varies in the range of $2-6 \%$, depending on the photon rate, i.e., the lower the photon rate, the higher the uncertainty. It was calculated according to the Guide to the Expression of Uncertainty in Measurement (GUM) [63]. The highest contribution is the statistical noise of the reference detector, which contributes to more than $90 \%$ of the overall uncertainty.

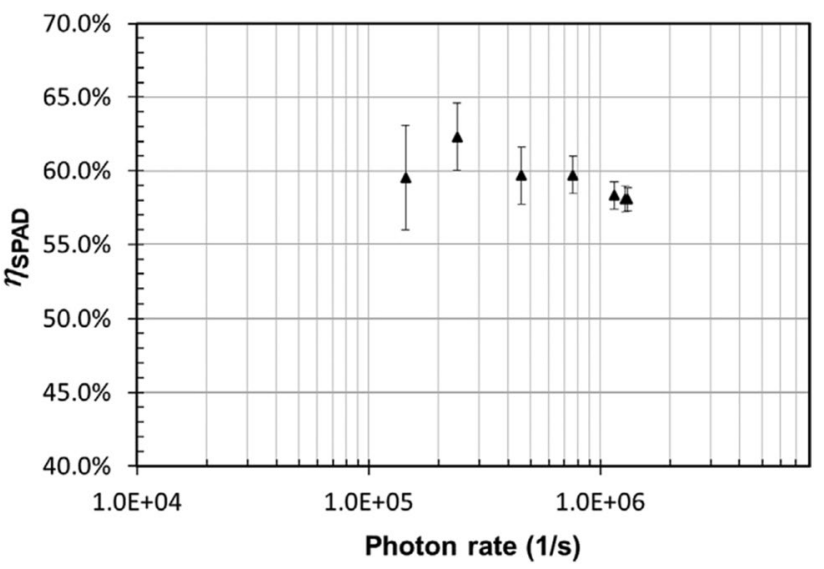

Fig. 3 Calibration result for the SPAD detection efficiency (Perkin Elmer, SPCM-AQRH-13-FC) using the molecule-based single-photon source and a low-noise reference analog detector. Taken from [58] 


\subsection{The InGaAs quantum dot based single-photon source for calibration of SPAD detectors}

Besides molecules and impurities centres in (nano-)diamond, also semiconductor quantum dots (QD) have been investigated for their use as sources for radiometric applications. QD based single-photon sources are well suited for this purpose, because of the possibility to operate them both continuous wave and triggered, they are robust, durable and photostable. Their emission spectrum has narrow bandwidth, and the wavelength of operation is usually in a range where the calibration of both $\mathrm{Si}$ - and InGaAs/InP-SPADs is possible. Furthermore, manufacturing processes, especially with respect to a designed dielectric environment for the QDs, are well advanced and improve constantly. The latter is of highest importance for achieving high extraction efficiencies and thus high photon fluxes. A first significant step towards the use of QD-based single-photon sources in radiometry was demonstrated in [64]. Georgieva et al. characterized an InGaAs QD-based single-photon source metrologically. The single-photon emitter consisted of an InGaAs QD, which was embedded into a monolithic microlens. This structure was positioned on top of a distributed Bragg reflector. With such a structure and using a collecting objective lens with a numerical aperture of 0.7 , an extraction enhancement of up to $23 \%$ is expected, see [65]. Additionally, an antireflection coating was applied on top of the microlens to further reduce collection losses. A detailed description of the fabrication process is given in [66]. The maximum photon flux obtained with that source was up to $3.7 \times 10^{5}$ photons per second at a wavelength of $(922.4 \pm 0.1) \mathrm{nm}$. The FWHM of the spectral line was determined to be $42 \mathrm{pm}$ and was limited by the spectrometer's resolution. The single-photon purity evaluated with the second-order correlation function at zero delay time, $g^{(2)}(\tau=0)$, was $0.24 \pm 0.06$. This value was limited

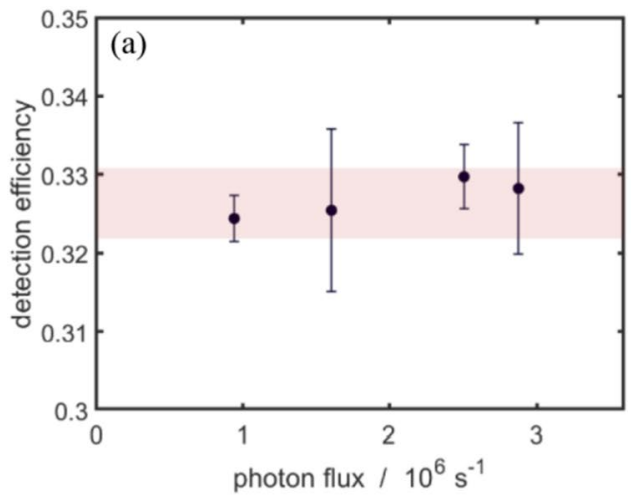

Fig. 4 Si-SPAD detector calibration. a Calibration using the spectrally filtered QD emission for a direct comparison with an analog reference detector. The pink area represents the expanded uncertainty $(k=2)$ of the weighted mean. b Calibration using a strongly attenuated laser source, where the incoming photon flux has been indirectly determined from a calibration of two variable attenuators. The error mainly by background emission from the sample matrix caused by non-resonant excitation and a long-living decay component. This source was then applied for the relative calibration of two Si-SPAD detectors against each other. An absolute calibration of the SPAD detectors directly against a conventional reference $\mathrm{Si}$-diode was not possible due to the low photon flux. However, the relative standard uncertainty was $0.7 \%$ and the obtained results were consistent with the ones obtained from the standard calibration method using an attenuated laser [9]. Also, an Allan deviation analysis was performed giving an optimal averaging time of $92 \mathrm{~s}$ for the photon flux.

In the subsequent work [67], a new structure of the QD sample was applied. The sample structure is grown by metal-organic chemical vapor deposition. Suitable QDs were selected by cathodoluminescence spectroscopy, whereas electron-beam lithography (EBL) is used to form micro-mesas at these pre-selected positions. These cylindrical shaped mesas with a radius between 600 and $640 \mathrm{~nm}$ have a height of approx. $800 \mathrm{~nm}$. For further information on the in-situ EBL nanotechnology process see [68]. Also, the efficiency of the whole setup was significantly improved by using optimized optical components. The maximum photon flux obtained was $(2.55 \pm 0.02) \times 10^{6}$ photons per second inside a multimode fibre at a wavelength of approximately $929.8 \mathrm{~nm}$. The non-resonant excitation occurred in a pulsed regime at a repetition rate of $80 \mathrm{MHz}$. The value of the second order correlation function $g^{(2)}(\tau=0)$ was between 0.14 and 0.24 , depending on the excitation power. With these parameters, this source is applicable for a Si-SPAD detector calibration directly against a calibrated, conventional reference detector, which is traceable to the national standard for optical radiant flux. Figure 4a depicts the results of the calibration. The apparent detection efficiency is shown as a function of the incoming photon flux. The lowest measurement

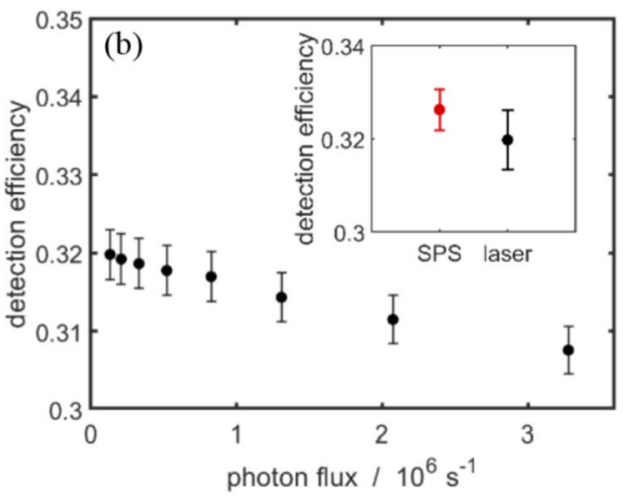

bars in (a) and (b) indicate the standard measurement uncertainty. Inset: comparison of the weighted mean of the DE from (a) with the $\mathrm{DE}$ from (b) for the lowest measured photon flux, where the error bars show the corresponding expanded uncertainties $(k=2)$. Taken from [67] 
Table 1 Summary of obtained results with single-photon sources applied for SPAD detection efficiency calibration

\begin{tabular}{llll}
\hline Type of source & $\begin{array}{l}\text { Impurity center in (nano-) } \\
\text { diamond }\end{array}$ & Single molecule & $\begin{array}{l}\text { Semiconductor quan- } \\
\text { tum dot }\end{array}$ \\
\hline Emitter & $\mathrm{NV}$ & DBT:Ac & InGaAs \\
$N_{\mathrm{ph}}($ photons/s) & $2.6 \times 10^{5}$ & $1.32 \times 10^{6}$ & $2.55 \times 10^{6}$ \\
$\Delta \lambda$ & $\approx 100 \mathrm{~nm}$ & $<0.2 \mathrm{~nm}$ & $<42 \mathrm{pm}$ \\
$N_{\mathrm{ph}, \lambda}(\lambda)($ photons/(nm s) $)$ & $\approx 1100$ & $1.32 \times 10^{6}$ & $2.55 \times 10^{6}$ \\
$g^{(2)}(\tau=0)$ & $(@ \approx 685 \mathrm{~nm})$ & $(@ 785.6 \mathrm{~nm})$ & $(@ 929.8 \mathrm{~nm})$ \\
$T(\mathrm{~K})$ & 0.23 & 0.08 & 0.24 \\
$u(\eta)$ & Room temperature & 3 & 10 \\
\hline
\end{tabular}

uncertainty obtained is approx. $1.2 \%$. The stability of the QD emission over time, the traceable calibration of the spectral sensitivity of the low-noise analogue detector and the change in the coupling loss of the fibre connector are the largest contributions to the overall uncertainty. The detection efficiency is, within the stated uncertainties, independent from the photon flux. The apparent detection efficiency has a weighted mean value (i.e., taking the uncertainties into account $)$ of $(32.63 \pm 0.22) \%$. For the traditional calibration using an attenuated laser, the measured detection efficiency is, on the contrary, strongly dependent on the incoming photon flux, see Fig. 4b. As expected, with increasing photon flux, a decrease of the apparent, measured detection efficiency is clearly observed. The photon statistics of the laser light together with the dead time of the Si-SPAD detector are responsible for this behaviour.

\section{Summary and conclusion}

In this paper, we reported on the metrological characterization of different types of single-photon sources and their application for the detection efficiency calibration of singlephoton detectors, which is a specific aspect within quantum radiometry and quantum metrology. Under investigation for this purpose are currently single-photon sources based on impurity centres in (nano-) diamond, single molecules and semiconductor quantum dots, both embedded in dielectric structures for collection efficiency enhancement. Table 1 summarizes the results obtained so far on the metrological characterization of single-photon sources, which is performed in a traceable way, with respect to photon flux, spectral power distribution, spectral photon flux and second order correlation $g^{(2)}(t)$, as well as the lowest uncertainties realized with these sources in detection efficiency calibration of SPAD detectors.

$N_{\text {ph }}$ : total photon flux, $\Delta \lambda$ : spectral bandwidth (FWHM), $N_{\mathrm{ph}, \lambda}(\lambda)$ : maximum spectral photon flux (normalized to a radiometric relevant bandwidth of $1 \mathrm{~nm}), g^{(2)}(t=0)$ : value of the second order correlation function at $t=0$ (indicator of single-photon purity, $T$ : operation temperature, $u(\eta)$ : uncertainty realized in SPAD detection efficiency calibrations.

As can be seen, best results thus far were obtained with the InGaAs/GaAs semiconductor quantum dot source with respect to all parameters. Next steps are the design and fabrication of optimized structures to further enhance the outcoupling efficiency and thus the single photon flux. Also, it is important to reduce the $g^{(2)}(t=0)$ value in order to avoid problems with multiphoton events in case of high photon rates. Still a drawback is the necessity to apply cryogenic temperatures. Also, for the single molecule DBT:Ac based source, very promising results were obtained. Further improvement is planned into the direction of pulsed operation, to avoid variation in photon arrival times or a Poissonian-like behaviour, as observed in the presented calibration results. It should also be noted that, thus far, dielectric structuring was not carried out for the molecule samples, so there is significant room for improvement. As for the semiconductor quantum dots, cryogenic temperatures are required for operation. With impurity centre-doped single-photon sources detection efficiency calibration could not be carried out, because of the low spectral photon flux in case of the nitrogen vacancy based single-photon source, resulting from the broadband emission. Whether other sources based on the silicon, germanium, tin and lead vacancy centres will exhibit sufficient spectral photon flux, while maintaining single-photon purity, is currently under investigation. As far as structuring in diamond is concerned, a lot of progress was realized using e.g., focused ion beam (FIB) and other techniques. The largest advantageous feature of diamond based single-photon sources is the possibility to be operated at room temperature.

However, it should be pointed out already, that the traceability gap between classical and quantum radiometry is closing with the help of the single-photon sources presenting in this paper. Further development will not only lead to even better results, i.e., lower uncertainties, but also to stable and robust operation. Also, within the EMPIR joint research project SEQUME (Single- and entangled photon sources for quantum metrology) [69, 70], further 
metrological applications using single-photon sources will be investigated.

Acknowledgements This work was funded by the project 17FUN06 SIQUST. This project has received funding from the EMPIR programme co-financed by the Participating States and from the European Union's Horizon 2020 research and innovation programme. This project (EMPIR 20FUN05 SEQUME) has received funding from the EMPIR programme co-financed by the Participating States and from the European Union's Horizon 2020 research and innovation programme. We gratefully acknowledge the support of the Braunschweig International Graduate School of Metrology B-IGSM and the DFG Research Training Group 1952 Metrology for Complex Nanosystems. This work was also supported by the Deutsche Forschungsgemeinschaft (DFG, German Research Foundation) under Germany's Excellence Strategy_EXC- 2123 QuantumFrontiers-390837967.

Funding Open Access funding enabled and organized by Projekt DEAL.

Open Access This article is licensed under a Creative Commons Attribution 4.0 International License, which permits use, sharing, adaptation, distribution and reproduction in any medium or format, as long as you give appropriate credit to the original author(s) and the source, provide a link to the Creative Commons licence, and indicate if changes were made. The images or other third party material in this article are included in the article's Creative Commons licence, unless indicated otherwise in a credit line to the material. If material is not included in the article's Creative Commons licence and your intended use is not permitted by statutory regulation or exceeds the permitted use, you will need to obtain permission directly from the copyright holder. To view a copy of this licence, visit http://creativecommons.org/licenses/by/4.0/.

\section{References}

1. M.D. Eisaman, J. Fan, A. Migdall, S.V. Polyakov, Single-photon sources and detectors. Rev. Sci. Instrum. 82, 071101 (2011)

2. A. Migdall, S. V. Polyakov, J. Fan, J. C. Bienfang, "Single-Photon Generation and Detection: Physics and Applications" Experimental Methods in the Physical Sciences, Volume 45, Hrsg. Academic Press, ISBN: 9780123876959, 2013.

3. N. Sangouard, H. Zbinden, What are single photons good for? J. Mod. Opt. 59, 1458 (2012)

4. C.J. Chunnilall, I.P. Degiovanni, S. Kück, I. Müller, A.G. Sinclair, Metrology of single-photon sources and detectors: a review. Opt. Eng. 53, 081910 (2014)

5. J.Y. Cheung, C.J. Chunnilall, E.R. Woolliams, N.P. Fox, J.R. Mountford, J. Wang, P.J. Thomas, The quantum candela: a redefinition of the standard units for optical radiation. J. Mod. Opt. 54, 373 (2007)

6. J.C. Zwinkels, E. Ikonen, N.P. Fox, G. Ulm, M.L. Rastello, Radiometry, photometry and the candela: evolution in the classical and quantum world. Metrologia 47, R15 (2010)

7. Mise en pratique for the definition of the candela and associated derived units for photometric and radiometric quantities in the SI, SI Brochure - 9th edition (2019) - Appendix 2 v1.02, Consultative Committee for Photometry and Radiometry, 22 March 2021

8. A. Sperling, S.T. Kück, The SI unit candela. Ann. Phys. 531, 5 (2019). https://doi.org/10.1002/andp.201800305

9. M. López, H. Hofer, S. Kück, Detection efficiency calibration of single-photon silicon avalanche photodiodes traceable using double attenuator technique. J. Mod. Opt. 62, S21-S27 (2015). https://doi.org/10.1080/09500340.2015.1021724

10. W. Schmunk et al., Radiometric calibration of single photon detectors by a single photon source based on NV-centers in diamond. J. Mod. Opt. 58, 1252 (2011)

11. X.L. Chu, S. Götzinger, V. Sandoghdar, A single molecule as a high-fidelity photon gun for producing intensity-squeezed light. Nat. Photon 11, 58-62 (2017). https://doi.org/10.1038/nphoton. 2016.236

12. B. Lounis, M. Orrit, Single-photon sources. Rep. Prog. Phys. 68 1129 (2005)

13. I.R. Berchera, I.P. Degiovanni, Quantum imaging with sub-Poissonian light: challenges and perspectives in optical metrology. Metrologia 56(2), 024001 (2019)

14. G. Brida, M. Genovese, I. Ruo Berchera, Experimental realization of sub-shot-noise quantum imaging. Nat. Photon. 4, 227 (2010)

15. N. Samantaray, I. Ruo-Berchera, A. Meda, M. Genovese, Realisation of the first sub shot noise wide field microscope. Light Sci. Appl. 6, e17005 (2017)

16. O. Schwartz, J.M. Levitt, R. Tenne, S. Itzhakov, Z. Deutsch, D. Oron, Superresolution microscopy with quantum emitters. Nano Lett. 13, 5832 (2013)

17 D. Gatto Monticone et al., Beating the Abbe diffraction limit in confocal microscopy via nonclassical photon statistics. Phys. Rev. Lett. 113, 143602 (2014)

18. S. Lloyd, Quantum illumination. Science 321, 1463 (2008)

19 E.D. Lopaeva et al., Experimental realization of quantum illumination. Phys. Rev. Lett. 110, 153603 (2013)

20. G. Petrini, E. Moreva, E. Bernardi, P. Traina, G. Tomagra, V. Carabelli et al., Is a quantum biosensing revolution approaching? Perspectives in NV-assisted current and thermal biosensing in living cells. Adv. Quantum Technol. 3(12), 2000066 (2020)

21. E. Moreva, E. Bernardi, P. Traina, A. Sosso, S.D. Tchernij, J. Forneris et al., Practical applications of quantum sensing: a simple method to enhance the sensitivity of nitrogen-vacancy-based temperature sensors. Phys. Rev. Appl. 13(5), 054057 (2020)

22 S. Pirandola, Quantum reading of a classical digital memory. Phys. Rev. Lett. 106, 090504 (2011)

23 G. Ortolano, E. Losero, I. Ruo Berchera, S. Pirandola, M. Genovese, Experimental quantum reading with photon counting. Sci. Adv. 7, eabc7796 (2021)

24. PTB-Mitteilungen. Band 130 (2020), Heft 3. ISSN 0030-834X. https://doi.org/10.7795/310.20200399

25. H. Georgieva, A. Meda, S.M.F. Raupach, H. Hofer, M. Gramegna, I.P. Degiovanni, M. Genovese, M. López, S. Kück, Detection of ultra-weak laser pulses by free-running single-photon detectors: modeling dead time and dark counts effects. Appl. Phys. Lett. 118, 174002 (2021). https://doi.org/10.1063/5.0046014

26. A. Gruber et al., Scanning confocal optical microscopy and magnetic resonance on single defect centers (PDF). Science 276(5321), 2012-2014 (1997). https://doi.org/10.1126/science. 276.5321 .2012

27. R.T. Harley, M.J. Henderson, R.M. Macfarlane, J. Phys. C 17, L233 (1984)

28. G. Davies, M.F. Hamer, Proc. R. Soc. Lond. A 348, 285-298 (1976)

29 C. Kurtsiefer, S. Mayer, P. Zarda, H. Weinfurter, Stable solid-state source of single photons. Phys. Rev. Lett. 85, 290 (2000)

30. M.W. Doherty, N.B. Manson, P. Delaney, F. Jelezko, J. Wrachtrup, L.C.L. Hollenberg, The nitrogen-vacancy colour centre in diamond. Phys. Rep. 528, 1 (2013)

31. Former company Quantum Communications Victoria. http://qcvic toria.com

32. B. Rodiek, M. López, H. Hofer, G. Porrovecchio, M. Šmid, X.-L. Chu, S. Götzinger, V. Sandoghdar, S. Lindner, C. Becher, S. Kück, Experimental realization of an absolute single-photon source 
based on a single nitrogen vacancy center in a nanodiamond. Optica 4, 71 (2017)

33 B. Rodiek, M. López, H. Hofer, S. Kück, The absolutely characterized nitrogen vacancy center-based single-photon sourcemeasurement uncertainty of photon flux and angular emission properties. J. Phys.: Conf. Ser. 972, 012008 (2018)

34. C.L. Wang, C. Kurtsiefer, H. Weinfurter, B. Burchard, Single photon emission from $\mathrm{SiV}$ centres in diamond produced by ion implantation. J. Phys. B 39, 37-41 (2006)

35. E. Neu et al., Single photon emission from silicon-vacancy centres in CVD-nano-diamonds on iridium. N. J. Phys. 13, 025012 (2011)

36. B. Pingault, D.D. Jarausch, C. Hepp, L. Klintberg, J.N. Becker, M. Markham, C. Becher, M. Atatüre, Coherent control of the siliconvacancy spin in diamond. Nat. Commun. 8, 15579 (2017)

37. A. Sipahigil, K.D. Jahnke, L.J. Rogers, T. Teraji, J. Isoya, A.S. Zibrov, F. Jelezko, M.D. Lukin, Indistinguishable photons from separated silicon-vacancy centers in diamond. Phys. Rev. Lett. 113, 113602 (2014)

38 P. Siyushev et al., Optical and microwave control of germaniumvacancy center spins in diamond. Phys. Rev. B 96, 081201 (2017)

39. D. Chen, Z. Mu, Y. Zhou, J.E. Fröch, A. Rasmit, C. Diederichs, N. Zheludev, I. Aharonovich, W. Gao, Optical gating of resonance fluorescence from a single germanium vacancy color center in diamond. Phys. Rev. Lett. 123, 033602 (2019)

40 T. Iwasaki et al., Tin-vacancy quantum emitters in diamond. Phys. Rev. Lett. 119, 253601 (2017)

41. S.D. Tchernij, T. Herzig, J. Forneris, J. Kupper, S. Pezzagna, P. Traina et al., Single-photon-emitting optical centers in diamond fabricated upon Sn implantation. ACS Photon. 4(10), 2580-2586 (2017)

42 M.E. Trusheim et al., Transform-limited photons from a coherent tin-vacancy spin in diamond. Phys. Rev. Lett. 124, 023602 (2020)

43. E. Corte, S. Sachero, S.D. Tchernij, T. Lühmann, S. Pezzagna, P. Traina et al., Spectral emission dependence of tin-vacancy centers in diamond from thermal processing and chemical functionalization. Adv. Photon. Res (2021). https://doi.org/10.1002/adpr.20210 0148

44. S. Ditalia Tchernij, T. Lühmann, E. Corte et al., Fluorine-based color centers in diamond. Sci. Rep. 10, 21537 (2020). https://doi. org/10.1038/s41598-020-78436-6

45 T. Lühmann et al., Screening and engineering of colour centres in diamond. J. Phys. D: Appl. Phys. 51, 483002 (2018)

46. G. Prestopino, M. Marinelli, E. Milani, C. Verona, G. VeronaRinati, P. Traina, E. Moreva, I.P. Degiovanni, M. Genovese, S. Ditalia Tchernij, F. Picollo, P. Olivero, J. Forneris, Photo-physical properties of He-related color centers in diamond. Appl. Phys. Lett. 111, 111105 (2017). https://doi.org/10.1063/1.4996825

47. S. Ditalia Tchernij, T. Luhmann, T. Herzig, J. Kupper, A. Damin et al., Single-photon emitters in lead-implanted single-crystal diamond. ACS Photonics 5(12), 4864-4871 (2018)

48 M.E. Trusheim et al., Lead-related quantum emitters in diamond. Phys. Rev. B 99, 075430 (2019)

49. S. Ditalia Tchernij, E. Corte, T. Lühmann, P. Traina, S. Pezzagna, I.P. Degiovanni et al., Spectral features of Pb-related color centers in diamond-a systematic photoluminescence characterization. New J. Phys. 23(6), 063032 (2021)

50. P. Wang, T. Taniguchi, Y. Miyamoto, M. Hatano, T. Iwasaki, Lowtemperature spectroscopic investigation of lead-vacancy centers in diamond fabricated by high-pressure and high-temperature treatment, http://arxiv.org/abs/2106.03413

51. E. Moreva, P. Traina, J. Forneris, S. Ditalia Tchernij, F. Picollo, I.P. Degiovanni, V. Carabelli, P. Olivero, M. Genovese, Color centres in diamond from single photon sources to ODMR in cells, Proceedings volume 10733. Quantum Photon. Dev. 2018, 1073304 (2018). https://doi.org/10.1117/12.2323102
52. A. Vaigu, G. Porrovecchio, X.-L. Chu, S. Lindner, M. Smid, A. Manninen, C. Becher, V. Sandoghdar, S. Götzinger, E. Ikonen, Metrologia, 2017, 54, 218; and erratum in Metrologia 2017, 54, 417.

53 J. Görlitz et al., NJP 22, 013048 (2020)

54 P. Fuchs et al., APL Photon. 6, 086102 (2021)

55. K. Lee, X. Chen, H. Eghlidi et al., A planar dielectric antenna for directional single-photon emission and near-unity collection efficiency. Nat. Photon 5, 166-169 (2011). https://doi.org/10.1038/ nphoton. 2010.312

56. A.A.L. Nicolet, B. Kozankiewicz, C. Hofmann, M. Orrit, ChemPhysChem 2007, 8 (1929)

57. C. Toninelli, K. Early, J. Bremi, A. Renn, S. Goetzinger, V. Sandoghdar, Opt. Express 18, 6577 (2010)

58. P. Lombardi, M. Trapuzzano, M. Colautti, G. Margheri, I.P. Degiovanni, M. López, S. Kück, C. Toninelli, A molecule based single photon source applied in quantum radiometry. Adv. Quantum Technol. (2019). https://doi.org/10.1002/qute.201900083

59. J.-B. Trebbia, P. Tamarat, B. Lounis, Phys. Rev. A 82, 063803 (2010)

60. S. Grandi, K.D. Major, C. Polisseni, S. Boissier, A.S. Clark, E.A. Hinds, Phys. Rev. A 94, 063839 (2016)

61. S. Pazzagli, P. Lombardi, D. Martella, M. Colautti, B. Tiribilli, F.S. Cataliotti, C. Toninelli, ACS Nano 12, 4295 (2018)

62. G. Porrovecchio, M. Šmid, M. López, H. Hofer, B. Rodiek, S. Kück, Comparison at the sub-100-fW optical power level between a high sensitive, low noise Silicon photodiode and a low optical flux measurement facility based on a double attenuator technique. Metrologia 53, 1115-1122 (2016). https://doi.org/10.1088/0026$1394 / 53 / 4 / 1115$

63. Guide to the Expression of Uncertainty in Measurement, 1st ed., BIPM, September 2008.

64. H. Georgieva, M. López, H. Hofer, J. Christinck, B. Rodiek, P. Schnauber, A. Kaganskiy, T. Heindel, S. Rodt, S. Reitzenstein, S. Kück, Radiometric characterization of a triggered narrowbandwidth single-photon source and its use for the calibration of silicon single-photon avalanche detectors. Metrologia 57(5), 055001 (2020). https://doi.org/10.1088/1681-7575/ab9db6

65. M. Gschrey et al., Highly indistinguishable photons from deterministic quantum-dot microlenses utilizing three-dimensional in situ electron-beam lithography. Nat. Commun. 6, 7662 (2015)

66. P. Schnauber et al., Bright single-photon sources based on antireflection coated deterministic quantum dot microlenses. Technologies 4, 1 (2016)

67. Georgieva, M. López, H. Hofer, N. Kanold, A. Kaganskiy, S. Rodt, S. Reitzenstein, S. Kück, Absolute calibration of a singlephoton avalanche detector using a bright triggered single-photon source based on a quantum dot. Opt. Express 29, 23500 (2021)

68. S. Rodt, S. Reitzenstein, High-performance deterministic in situ electron-beam lithography enabled by cathodoluminescence spectroscopy. Nano Express 2, 014007 (2021)

69. https://sequme.cmi.cz/

70. https://www.euramet.org/research-innovation/search-researchprojects/details/project/single-and-entangled-photon-sources-forquantum-metrology/?L=0\&tx_eurametctcp_project $\% 5$ Baction $\%$ $5 \mathrm{D}=$ show\&tx_eurametctcp_project $\% 5 \mathrm{~B}$ controller $\% 5 \mathrm{D}=$ Project $\&$ $\mathrm{cHash}=215 \mathrm{cdb} 43120 \mathrm{fadc5} 424 \mathrm{a} 182 \mathrm{c} 4 \mathrm{ba} 67589$

Publisher's Note Springer Nature remains neutral with regard to jurisdictional claims in published maps and institutional affiliations. 